\title{
Digital Object Memories for the Internet of Things (DOMe-loT)
}

\author{
Alexander Kröner ${ }^{1}$, Jens Haupert ${ }^{1}$, Chris Speed ${ }^{2}$, \\ Fahim Kawsar ${ }^{3}$, Thomas Ploetz ${ }^{4}$, and Daniel Schreiber ${ }^{5}$ \\ ${ }^{1}$ German Research Center for Artificial Intelligence (DFKI), Germany \\ ${ }^{2}$ Edinburgh School of Architecture and Landscape Architecture, Scotland \\ ${ }^{3}$ Bell Labs, Alcatel-Lucent, Belgium \\ ${ }^{4}$ School of Computing Science, Newcastle University, Newcastle upon Tyne, UK \\ ${ }^{5}$ Telecooperation Lab, Technische Universität Darmstadt, Germany
}

\begin{abstract}
Digital Object Memories (DOMe) comprise hardware and software components, which together provide an open and universal platform for capturing and interacting with the digital information of connected objects - including storage, documentation and provision of information concerning actions an object is or might be involved in. We envisage that connected objects will be enabled to make suggestions and propositions to human users - which implies that an object may have a level of agency. The latter concept is a striking possibility that may change the way that we perceive, interact and relate to objects. The goal of this established workshop series is to twofold: 1.) initiate a conversation concerning the potential for objects to develop agency; and 2.) explore how data that is associated with an object may leverage real-world actions. DOMe-IoT 2012 provides a hybrid interdisciplinary workshop format that will combine traditional presentations and discussion with practice-based experimentation.
\end{abstract}

\section{Author Keywords}

Ubiquitous Computing, Digital Object Memories, Internet of Things, Object-Centered Information Management, Agency.

\section{ACM Classification Keywords}

C2.4 [Computer-Communication Networks] Distributed Systems, H.3.2 [Information Storage and Retrieval] Information Storage, H.5 [Information Interfaces and Presentation], I.2.11 [Artificial Intelligence] Distributed Artificial Intelligence, J.7 [Computers in Other Systems].

Permission to make digital or hard copies of all or part of this work for personal or classroom use is granted without fee provided that copies are not made or distributed for profit or commercial advantage and that copies bear this notice and the full citation on the first page. To copy otherwise, or republish, to post on servers or to redistribute to lists, requires prior specific permission and/or a fee.

UbiComp' 12, Sep 5 - Sep 8, 2012, Pittsburgh, USA.

Copyright 2012 ACM 978-1-4503-1224-0/12/09...\$10.00.

\section{General Terms}

Design, Experimentation, Human Factors.

\section{INTRODUCTION}

Ubiquitous computing is instigating a transformation of our environment into an information sphere spread across time and space by combining sensing and software services with physical world. This essentially creates novel design opportunities for everyday objects, building blocks of our environment as their behavior are determined by software and affords us to build imaginative new forms of interaction and functionalities that dramatically enhances their well established features. Our physical space now represents an ecological synergy of networked smart objects tagged with awareness technologies - computational memory, sensors and actuators. This unfolds a range of imaginative possibilities to discover, manage, compose, coordinate, and control physical space to realize personalized and coordinated behavior within and across devices and provide the foundation for the Internet of Things.

For the past years the workshop Digital Object Memories for the Internet of Things (DOMe-IoT) has nurtured a theoretical and practical vocabulary across cultural contexts as well as technical applications. The exchange of experiences and insights across what remains an emerging technology is beginning to offer a critique of an IoT that has previously been logistical, and in its place an understanding for the platform that values a social dimension. The purpose of DOMe-IoT remains in bringing together researchers from a variety of disciplines (computer scientist, electrical engineers, designers, social scientists, etc.) to discuss various aspects of Digital Object Memories and its significance with respect to IoT applications.

DOMe-IoT continues an established workshop series [21]; predecessor events include DIPSO 2007-09 in conjunction with Ubicomp 2007-09, DOMe in conjunction with Intelligent Environment 2009, DOMe-IoT 2010 in conjunction with Ubicomp 2010, and NOMe-IoT in conjunction with Ubicomp 2011. 


\section{WORKSHOP TOPICS}

In 2012, suggested topics for submissions to the workshop include (but are not limited to):

- Objects and Agency: As the databases that are connected to objects begin to exchange data, the correlations and analysis of historical data will lead to predictive models that will be articulated through objects. In these contexts things will begin to make suggestions and propositions to human users. Depending upon their sophistication these 'commands' imply that an object may have a level of agency. The concept that a thing may have agency is a striking possibility that may change the way that we further relate to objects.

- Architectures: General architectures and middleware approaches which allow for the realization of object memory functionality for IoT. This includes infrastructures for the centralized or distributed capturing, organizing, storing, and exploiting of object-related information, directly on the physical object itself or based on some remote infrastructure.

- Memory Content Representation and Modeling: Formats for memory content items, discussion of standards and best-practice knowledge concerning the representation of object-related knowledge.

- Memory Creation: Technologies and concepts for the manual, semi-automatic, or automatic creation of whole memories or single memory entries. This includes physical sensor readings, information inferred from external sources, and user-generated content.

- Data Mining: Information stored in a digital object memory might be analyzed in order to discover typical usage patterns or anomalies. Such information might help human users or other environments to better deal with smart items.

- Interaction with an Object Memory: This topic comprises technologies and concepts to make an object memory's content accessible to human users. One of the major challenges here is how to structure, relate, prepare, and explain the wide variety of diverse data that might be contained in the memory due to its open nature.

- Applications: Novel application scenarios of digital object memories for IoT and existing prototypes.

- Privacy and Legal Aspects: Who "owns" the data stored in memory of an IoT, who can access/delete/correct it? How long must/should memory content be stored, and how can trust be established for the object memory?

- Social Implications: Object memories have the potential to change the way we perceive our surrounding. Not only humans now can tell their personal story, but every object potentially allows us to investigate its history and understand how our world is connected. This might fundamentally influence our relation to objects and other humans, too.
In 2012, the workshop will focus on research issues related to agency and object memories. Here, the term "agency" makes reference to Actor-Network Theory (ANT). The latter one can be seen as a tool for exploring and describing how the social is assembled by way of technologies, objects and artifacts. The theory distributes agency, specifically responsibility, equally across entities within the network, including a host of nonhuman ones not normally seen as exercising agency at all [25]. ANT does not typically attempt to explain why a network exists; it is more interested in the infrastructure of actor-networks, how they are formed, maintained and how they can fall apart. ActorNetwork Theory incorporates what is known as a principle of generalized symmetry; that is, what is human and nonhuman (e.g. artifacts, organization structures) should be integrated into the same conceptual framework and assigned equal amounts of agency. An actor is not the source of action but the moving target of a vast array of entities swarming towards it, and action should be felt as a set of agencies or translations between mediators that may generate traceable associations.

In the Internet of Things, "agency happens with the ecology of networked public media - streams, feeds, trackbacks, permalinks, Wiki inscriptions and blog posts" [2]. In other words the agency lies in the flow of data between networked objects. DOMe-IoT 2012 wants to devote special attention to implications of this observation on communication between and with networked object memories. Presentations and discussions at previous workshops revealed a potential for object memories to document processes as well as the actions and experiences of users. This iterative and complex interrelationship between data, correlation and action requires investigation into the actual performance of such digital records.

Following this general direction, DOMe-IoT 2012 will devote special attention to questions related to the modeling of agency for object memories, combining physical artifacts and their digital representation with services in the virtual and actors in the real world, how to identify actions (tasks) supported by an object and its memory, and effects (added value as well as user experience) resulting from this approach. The workshop at UbiComp 2012 will allow participants to discuss and explore the implications of a future in which a home appliance may use its memory in order to reason about the way it is used - and take over responsibility for its own behavior.

\section{RELATED LITERATURE}

Research on smart objects and the Internet of Things has been going on for more than a decade and reaches back to Mark Weiser's original vision of ubiquitous computing. Bruce Sterling recently popularized the idea of smart objects and the Internet of Things; Sterling coined the term Spime [1] to describe a new category of space-time objects that are aware of their surroundings and can memorize real world events. Julian Bleeker advocated a similar notion of 
blogjects (objects that blog) in his "Manifesto for Networked Objects" [2].

This more visionary work has been met by a growing body of technology- and business-focused research on RFID, smart objects, and smart products [3]. For instance, the electronic pedigree [17] aims at protecting consumers from contaminated medicine or counterfeit drugs. It establishes a link between physical item and external data sources which enables a verification of the physical item's integrity along several steps of such products' lifecycle. Such integrityoriented solutions may get further support if objects may contribute to the data collection process with own sensing and processing capabilities - as suggested by Decker et al. for object-based tracking and quality monitoring in logistics [22].

The aforementioned applications rely on a continuous linking of data along a process involving several entities (e.g., business partners). In order to ease building and exploiting such data collections, Schmitt et al. [19] emphasize the value of open structures for data collection and exchange. This is also reflected by the approach of Schneider [21]. He proposes a Web-based Object Memory, which serves as an open reference to object-related information hosted and controlled by arbitrary content providers.

Roy Want and his colleagues augmented physical objects with passive RFID tags so that they were uniquely identifiable and information related to them could be presented to their users [4]. Michael Beigl and his colleagues defined a smart object as an everyday artifact augmented with computing and communication, enabling it to establish and exchange information about itself with other artifacts and/or computer applications" [5]. Friedemann Mattern formulated in a similar way: "Smart" objects might be able to not only to communicate with people and other smart objects, but also to discover where they are, which other objects are in the vicinity, and what has happened to them in the past" [6]. Norbert Streitz and his colleagues looked at smart objects from two perspectives: one model has system-oriented, importunate smartness in which smart objects can take certain self directed actions based on previously collected information; the other is people-oriented, empowering smartness where smart objects empower users to make decisions and take mature and responsible actions [7]. Both models include questions related to agency and responsibility. For instance, a "smart product" can be enabled to take over responsibility for a defined step of a larger business process [20] - or employ annotated workflows embedded into their object memory in order to continuously take action during a complex process [24].

Most recent work on smart objects has focused on technical aspects (e.g., hardware platforms, software infrastructure [8]) and application scenarios. Application areas range from supply chain management and enterprise applications [9] to (home and hospital) healthcare [9] and industrial workplace support [10-14]. Human-interface aspects of smart-object technology are just beginning to receive attention [15]. Yet design principles and methods for smart objects that go beyond mere hardware have yet to be explored (see also Fahim Kawsar's dissertation [16]). Regarding agency, examples from related work already illustrate potential benefits from objects with associated data collections on human behavior - for instance, in support of mindful eating [23] or therapy compliance [27].

Other works are related to the issue of modeling data for and structure of object memories. For instance, with the increasing distribution of smart labels in mind, Maass and Filler [18] suggest to link physical product items with digital information in order to support customer-oriented services such as a product comparison. They rely on a limited set of facets (e.g., "business", "security") to encode domain-dependent views on a product. For open and flexible storage of object memories in web-based structures, the W3C Object Memory Modeling Incubator Group proposes an Object Memory Format, which combines standardized memory contents in a block-based container [26].

\section{EXPECTED OUTCOME}

DOMe-IoT seeks to provide participants with a "hands-on" experience of Digital Object Memories. Participants will introduce their research concerning digital object memories in position statements, and attend a practical exercise concerning the workshop's focus topic "agency and digital object memories".

We expect three types of results from the proposed workshop:

1. Submitted position papers and a documentation of the group discussions (slides) will be provided on the workshop homepage. Furthermore, we will consider contacting a publisher for a special journal issue or book series volume considering the quality and novelty of the position papers.

2. Design case studies will be solicited as an explicit submission category for the workshop. From our previous experience we anticipated that such a category will be particularly attractive to designers and digital media artists. Contributions from creative practice disciplines have significant potential to stimulate discussion around nontraditional notions of object memory. Post-workshop, accepted designs and the workshop discussion will be made available on the workshop website.

3. We also plan to conduct a live experiment at UbiComp, in which workshop participants transform physical objects into smart artifacts supporting agency - concerning memory contents, digital services, and actions related to the object. Afterwards, participants' feedback will be collected and exploited to leverage discussion concerning expectations towards agency of smart artifacts. As outcome of this discussion, a set of agency-related functions of object 
memories should be defined. We plan to present a preliminary evaluation of this utilization of object memories through the UbiComp participants at the third conference day (e.g. in form of a poster and/or a short presentation). We furthermore plan to document the live experiment on the workshop website [21] and use this to promote a sustained interaction between the participants after UbiComp.

\section{ACKNOWLEDGEMENT}

We thank the DOMe-IoT program committee members for contributing to the success of the workshop with their time and expertise. We also thank the Ubicomp 2012 organizers for providing us with the opportunity and infrastructure to run the DOMe-IoT 2012 workshop. This research was funded in part by the German Federal Ministry of Education and Research under grant number 01IA11001. The responsibility for this publication lies with the authors.

\section{REFERENCES}

1. Sterlings, B. Shaping Things, MIT Press (2005).

2. Bleecker, J. A Manifesto for Networked Objects Cohabiting with Pigeons, Arphids and Aibos in the Internet of Things. Near Future Laboratory (2006), http://nearfuturelaboratory.com/files/WhyThingsMatter. pdf

3. Thiesse, F., and Kohler, M. An Analysis of UsageBased Pricing Policies for Smart Products. In Electric Markets 18, 3, Routledge (2008), 232-241.

4. Want, R. et al. Bridging Physical and Virtual Worlds with Electronic Tags. In Proc. ACM SIGCHI 99, ACM Press (1999), 370-377.

5. Beigl, M., Gellersen, H.-W., and Schmidt, A. MediaCups: Experience with Design and Use of Computer-Augmented Everyday Objects. In Computer Networks 35, 4, Elsevier (2001), 401-409

6. Mattern, F. From Smart Devices to Smart Everyday Objects. In Proc. SmartObjects Conf. (SOC 03), Springer (2003), 15-16.

7. Streitz, N., et al. Designing Smart Artifacts for Smart Environments. In Computer 38, 3, IEEE (2005), 41- 49.

8. Siegemund, F. A Context-Aware Communication Platform for Smart Objects". In Proc. 2nd Int'l Conf. Pervasive Computing (PERVASIVE 04), Springer (2004), 69-86.

9. Konomi, S., and Roussos, G. Ubiquitous Computing in the Real World: Lessons Learnt from Large-Scale RFID Deployments. In Personal and Ubiquitous Computing 11, 7, Springer (2007), 507-521.

10. Strohbach, M., et al. Cooperative Artefacts: Assessing Real World Situations with Embedded Technology. In
Proc. Int'l Conf. Ubiquitous Computing (Ubicomp 04), Springer (2004), 250-267.

11. Kortuem, G. et al. Sensor Networks or Smart Artifacts? An Exploration of Organizational Issues of an Industrial Health and Safety Monitoring System. In Proc. Int'l Conf. Ubiquitous Computing (Ubicomp 07), Springer (2007), 465-482.

12. Fitton, D. et al. Exploring the Design of Pay-Per-Use Objects in the Construction Domain. In Proc. $3^{\text {rd }}$ European Conf. Smart Sensing and Context, Springer (2008), 192-205.

13. Efstratiou, C. et al. Experiences of Designing and Deploying Intelligent Sensor Nodes to Monitor HandArm Vibrations in the Field. In Proc. 5th Int'l Conf. Mobile Systems, Applications, and Services (MobiSys 07), ACM Press (2007), 127-138.

14. Kortuem, G., Kawsar, F., Fitton, D., and Sundramoorthy, V. Smart Objects as Building Blocks for the Internet of Things. In IEEE Internet Computing, Special Issue of Internet of Things 14, 1, IEEE (2010), 44-51.

15. Nelson, L., and Churchill, E.F. User Experience of Physical-Digital Object Systems: Implications for Representation and Infrastructure. In Proc. of Smart Object Systems Workshop, in conjunction with Int'1 Conf. Ubiquitous Computing (Ubicomp 05), 2005.

16. Kawsar, F. A Document-Based Framework for User Centric Smart Object Systems. PhD dissertation, Dept. Computer Science, Waseda Univ., Feb. 2009.

17. EPCglobal, Pedigree Ratified Standard 1.0., EPCglobal (2007), http://www.epcglobalinc.org/standards/pedigree

18. Maass, W., and Filler, A. Tip 'n Tell: Product-Centered Mobile Reasoning Support for Tangible Shopping. In Proc. of MSWFB 2007: Making Semantics Work For Business, part of 1st European Semantic Technology Conference, Vienna, Austria, 2007.

19. Schmitt, C. et al. Towards Ambient Business: Enabling Open Innovation in a World of Ubiquitous Computing. In Advances in Ubiquitous Computing: Future Paradigms and Directions, Idea Group Inc, Hershey (2008).

20. Decker, C. et al. Collaborative Business Items. In Proc. of the 3rd Int'l Conference on Intelligent Environments (IE 07), IEEE (2007), 40-47.version of this document that we modified. Some of the references cited in this paper are included for illustrative purposes only.

21. Kröner, A. et al. Homepage of "Digital Object Memories for the Internet of Things". DFKI (2012), http://www.dfki.de/dome-workshop/2012/ 